\title{
Ultrasound imaging of forearm fractures in children: a viable alternative?
}

\author{
Dominic Williamson, Roland Watura, Mark Cobby
}

\begin{abstract}
Objective-A pilot study to investigate whether ultrasonography can be reliably used to demonstrate uncomplicated greenstick and torus fractures in children. Method-Children between the ages of 2 and 14 years with a high clinical suspicion of a non-articular, undisplaced forearm fracture were included. Ultrasound imaging of the injury was performed by a consultant radiologist who gave an immediate report. Standard radiographs of the forearm were then obtained and the patient treated in the normal way. The radiograph was formally reported on at a later date. Results-26 patients were included. There was an absolute correlation between the ultrasound and radiographic findings. The procedure was well tolerated.

Conclusion-Ultrasound seems effective for detecting uncomplicated forearm fractures in children. The procedure is easy to perform and the images easy to interpret. A larger study will now be undertaken to confirm these initial findings.

(F Accid Emerg Med 2000;17:22-24)
\end{abstract}

Keywords: ultrasound; fracture; forearm; children

The hospital assessment of children with injuries presents a particular problem. They are often in pain, scared and intimidated by their surroundings. Anxious parents often compound the fears of their children. Emergency departments across the country are trying to tackle this issue by creating specialised areas within the department and specifically trained staff to care for children. ${ }^{1}$ Such interventions limit their movement around the hospital and minimise exposure to other patient groups. However, problems can arise when children are required to go to other parts of the hospital or departments for further investigations, including radiology.

Although exposure to high dose ionising radiation is known to be carcinogenic and teratogenic, the harmful effects of low dose ionising radiation are debated. ${ }^{2}{ }^{3}$ However, it is a widely held view that the effects of exposure are cumulative and may contribute to the development of significant problems over a lifetime. Where possible the use of radiographic imaging should be avoided, especially in respect to children, but also their parents (adults of child bearing age) and staff. This study explores the possibility of routinely using ultrasound imaging for long bone fracture detection in children, with the aim of reducing overall exposure to ionising radiation.

Diagnostic ultrasonography has been well researched but evidence of its use in bony imaging and fracture detection is limited. Therapeutic ultrasound, with its potential to cause pain at a fracture site has proved unreliable in detecting fractures, ${ }^{45}$ especially in children. ${ }^{6}$ Case reports provide evidence that ultrasound imaging can be used in the detection of occult fractures. ${ }^{78}$ Two studies have shown a good correlation between ultrasound imaging and radiographic findings in new borns and cadavers. ${ }^{910}$ In some centres sonography has been reliably used to detect growth plate injuries, ${ }^{11}$ but this practice is not widespread.

In theory imaging with ultrasound should be easy to perform and yield good results. Bone is a natural obstacle to the transmission of sound at high frequency. The large difference in acoustic impedance at the junction of soft tissue and bone causes almost total reflection of sound energy providing a strong image of the bone surface. Any imperfections (for example, steps, breaks, etc) should therefore be easily visible. This technique is particularly applicable in children given their comparatively small soft tissue thickness and its non-invasive nature.

\section{Method}

Ethical approval for this study was obtained from the local ethics committee. Children between the age of 2 and 14 years with a suspected isolated, undisplaced, non-articular forearm fracture were asked to participate. Children were excluded if there was any clinical evidence of deformity. Each child was assessed by the emergency department staff grade, specialist registrar or associate specialist to determine whether a radiograph was indicated. As there is no validated system of determining the need for further investigation, patients were included on the basis of the attending physician's clinical suspicion. Factors that were considered included the mechanism of injury, the presence of pain, or swelling, or bony tenderness, or all three, related to either the radius or ulna. Clinical findings were recorded on a formal results sheet. Consent was obtained from the attending parent/ guardian and patient if appropriate. All subjects attended the emergency department at Frenchay Hospital, Bristol over a 12 month period from July 1997.

Using an ATL 2000 machine (ATL Ultrasound, Bothell, USA) with a $10 \mathrm{MHz}$ linear array probe and a musculoskeletal (hand/wrist) 


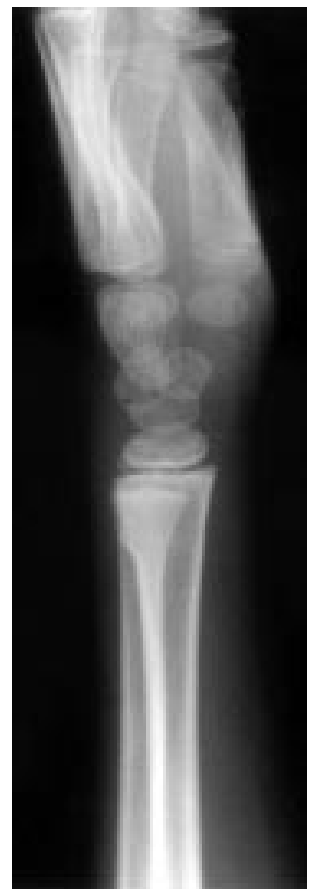

Figure 2 A radiographic image of the same injury as figure 1 .

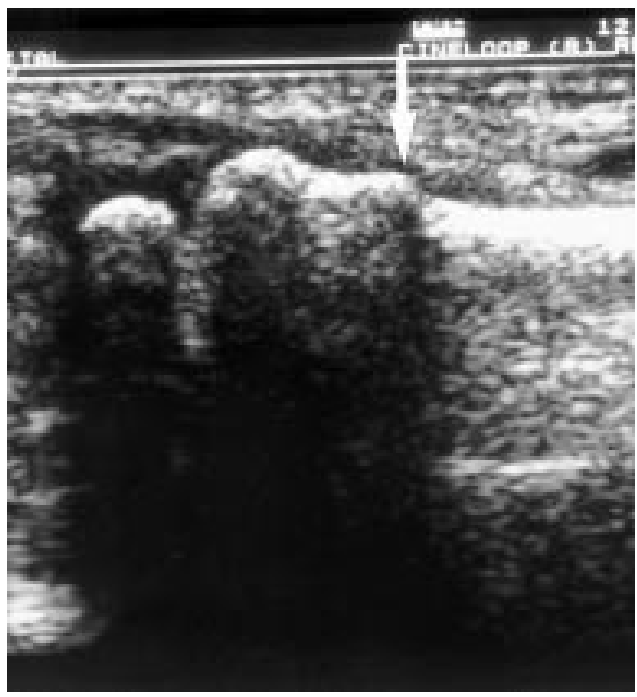

Figure 1 An ultrasound image of a greenstick fracture of the distal radius (arrow).

protocol, ultrasound of the injured forearm was performed by one of two consultant radiologists and the results recorded. All scans were performed in the General X-ray Department at Frenchay Hospital before any radiographic investigation. During the procedure each patient was specifically asked whether they were experiencing any additional pain or discomfort and their response recorded.

Standard two view radiographs of the forearm were then taken. Hard copies of both ultrasound and radiographic images were made and the results of the radiograph recorded. The radiographs were formally reported on at a later date as per departmental policy. This report was then compared with the initial ultrasound findings.

Each child was treated in the most appropriate way according to their injury, as determined by their clinical and radiogaphic findings. There was no specific patient follow up required for trial purposes.

\section{Results}

Twenty six children were included (10 girls and 16 boys) between the ages of 2 to 13 years (mean 8 years). Sixteen fractures were detected with ultrasound, all were confirmed at radiography (fig 1 and fig 2). All children who had normal ultrasound findings also had normal radiographs despite symptoms and clinical findings that were suspicious of a fracture. No bony abnormalities were detected by ultrasound that were not seen on the radiographs.

In this series the sensitivity and specificity for ultrasound detection of fractures was $100 \%$.

There were no reports of discomfort using the $10 \mathrm{MHz}$ imaging probes in this series.

The documented clinical findings indicating the requirement for further imaging was consistent for all patients.

\section{Discussion}

These results are based on a relatively small number of patients. However, they do suggest that ultrasound is as good as radiography in the detection of isolated, uncomplicated forearm fractures in children.

Previous trials using ultrasound to detect fractures have used therapeutic rather than imaging frequencies, that is $1 \mathrm{MHz}$ rather than $3.5 \mathrm{MHz}$ or greater. Therapeutic wavelengths often, but not consistently, elicit pain at the fracture site. ${ }^{4-6}$ There were no reports of pain or discomfort while using the $10 \mathrm{MHz}$ probe over the fracture site.

As a result of careful patient selection and clinical examination, there were no children with significant angulation or displacement about a fracture, as subsequently detected by radiography. No child required a manipulation. In fact, it is the investigators' perception that the degree of cortical deformation is more obvious with ultrasound than with radiography.

With increased awareness over the dangers of radiation, even at small doses, alternatives to radiography should be sought. The use of ultrasound imaging in all aspects of diagnostic medicine is rising. It is thought to be safe, reliable, and is being increasingly used in emergency medicine. ${ }^{12}$ High quality ultrasound imaging equipment is usually mobile, is becoming easier to use and produces very clear images. In this study fracture recognition using ultrasound proved to be very simple. With minimal training emergency clinicians should be able to perform the procedure themselves.

As a viable alternative to radiography, it may be feasible to move the diagnosis of simple bony injuries into the emergency department. This would not only reduce the use of ionising radiation, but also waiting times for children. It would make it unnecessary for them to leave the department for radiography, limit the number of people having to attend the child, and reduce the workload of radiology staff. A correct diagnosis could be made, management plans formulated and the child and parents advised accordingly, while the child remains in one designated room. Overall times in the hospital could be shortened and potential stress factors eliminated, all without irradiating the child.

From an orthopaedic point of view, the use of ultrasound imaging should not effect the subsequent management of a greenstick fracture. In this series, probably because of careful patient selection, there was no child with a significant degree of angulation about the fracture site (that is $>5^{\circ}$ as detected by radiography). In each case the fracture was clearly demonstrated by ultrasound. Indeed the clarity of the image is such that the authors believe that any clinically unsuspected angulation would easily be seen and therefore measured using this technique. Obviously this would need to be validated by further studies.

Recruitment into this trial was hampered by limited availability of resources. A more substantial trial with consecutive recruitment using dedicated equipment and staff would obviously improve the significance of the results. Data collection on such a trial is ongoing, with an emergency physician, having 
received approximately one hour training, performing all scans.

This pilot shows that using ultrasound imaging for the initial diagnosis of simple long bone fractures seems to be a viable alternative to radiography that warrants further investigation.

Conflict of interest: none.

Funding: none.

Contributors

Dominic Williamson, the principal investigator, initiated the research, developed the core idea, designed the protocol, participated in data collection, analysed the data and wrote the paper. Mark Cobby and Roland Watura helped in the study design, data collection and edited the paper. Paul Younge helped develop the core idea. Jason Kendall helped in the study design and was generally encouraging. The staff at Frenchay Hospital Emergency Department helped in the data collection.

1 Bentley J. Child-related services in general accident and emergency departments. F Advan Nurs 1996;24:1184-93.

2 Borja-Aburto VH, Bustamante-Montes P, Garcia-Sancho $\mathrm{MC}$, et al. [Ionising radiation at low doses and cancer: epidemiological controversy]. [In Spanish] Rev Invest Clin 1990;42:312-16.
3 Grazer RE, Meislin HW, Westerman BR, et al. A nine year evaluation of emergency department personnel exposure to onising radiation. Ann Emerg Med 1987;16:340-2.

4 Shenouda NA, England JP. Ultrasound diagnosis of scaphoid fractures. F Hand Surg Br 1987;12:43-5.

5 DaCruz DJ, Taylor RH, Savage B, et al. Ultrasound assessment of suspected scaphoid fracture. Arch Emerg Med 1988;5:97-100

6 Moss A Mowat AG Ultrasonic assessment of stress fractures. BM7 1983;286:1949.

7 Graif M, Stahl-Kent V, Ben-Ami T, et al. Sonographic detection of occult bone fractures. Pediatr Radiol 1988;18: 383-5.

8 Hunter JD, Mann CJ, Hughes PM. Fibular fracture: detection with high-resolution diagnostic ultrasound. F Accid Emerg Med 1998;15:118.

9 Katz R, Landman J, Dulitzky F, et al. Fracture of the clavicle in the newborn. An ultrasound diagnosis. F Ultrasound Med 1988;7:21-3.

10 Grechenig W, Clement HG, Fellinger M, et al. Scope and limitations of ultrasonography in the documentation of fractures-an experimental study. Arch Orthop Trauma Surg 1998;117:368-71.

1 Stewart J, Boyd R. Ultrasound scans and MRI of skeletal injuries in children. The AE $E$ Letter 1996;6:7.

12 Stahmer SA. Recent advances: accident and emergency medicine. BMF 1998;316:1071-4. 